\title{
A MONOLITHIC POLYDIMETHYLSILOXANE PLATFORM FOR ZOOSPORE CAPTURE, GERMINATION AND SINGLE HYPHA FORCE SENSING
}

\author{
Yiling Sun ${ }^{1}$, Ayelen Tayagui ${ }^{1,2}$, Ashley Garrill ${ }^{2}$, and Volker Nock ${ }^{1}$ \\ ${ }^{1}$ Electrical and Computer Engineering, University of Canterbury, Christchurch, NEW ZEALAND and \\ ${ }^{2}$ School of Biological Sciences, University of Canterbury, Christchurch, NEW ZEALAND
}

\begin{abstract}
This paper reports a triple-layer, polydimethylsiloxane (PDMS)-based lab-on-a-chip platform combining the capture and culture of individual oomycete zoospores with integrated force sensing on germinated hyphae. The platform enables the concurrent study of cell-to-cell variability in hyphal growth and protrusive force generation. To demonstrate the applicability of the platform, individual zoospores of the oomycete Achlya bisexualis were trapped by a constriction structure, cultured on the device and the micro-Newton forces exerted by hyphae measured by tracking the deflection of elastomeric micropillars. The platform provides a new tool to help understand protrusive growth on a single cell level.
\end{abstract}

\section{KEYWORDS}

Lab-on-a-Chip, Single Cell Analysis, Membrane Valve, Force Sensor, Fungi and Oomycetes.

\section{INTRODUCTION}

Hyphal microorganisms, such as fungi and oomycetes, can act as pathogens and cause significant threat to both plants and animals [1]. Several species actively impact food security and primary industries by attacking crops [2] and forestry plants [3], both which can lead to major economic losses. The spread of these diseases is further compounded by climate change [4], emergence of drug resistance [5] and world-wide dispersion by the globalization of human activities [6]. Recent years have seen increasing efforts to screen naturally bioactive compounds and develop new biocontrol strategies alternative to agrochemicals [7].

Common to all these activities is the need to screen a large number of potential candidates for their impact on fungal and oomycete growth and penetrative force generation at various stages in their respective life cycles. One such crucial stage is the germination of hyphae from either zoospores or conidia, leading to penetration into and infection of parts of a plant or animal [8]. This capability of tips growing from fungal hyphae to penetrate throughout host tissue is essential for pathogenicity [8]. The process is driven by cytoskeletal changes [9] and the yielding of hyphal tip to the internal hydrostatic pressure (turgor), which manifests externally as the generation of a protrusive force [10].

To better understand protrusive force generation by hyphal organisms, we have demonstrated force sensing technology based on elastomeric micropillars [11]. We used this technology to measure forces exerted by hyphae of the oomycete Achlya bisexualis [12] and the fungi Neurospora crassa [13] growing from mycelia seeded into monolithic polydimethylsiloxane (PDMS)-based devices. In the current work we extend on this by combining our in-channel high-aspect ratio sensing pillars for protrusive force measurements on hyphal organisms [14] with zoospore trapping, on-chip germination and maintenance [15]. This allows for high-throughput compartmentalization of individual zoospores or conidia into separate force measurement channels for parallelized screening, taking into account cellular heterogeneity, nuclear distribution and dynamics at the sub-hyphal level in response to exposure to various biocontrol strategies.

\section{EXPERIMENTAL \\ Platform design}

The monolithic PDMS platform consists of two layers separated by a membrane of $15 \mu \mathrm{m}$ thickness, as shown in Fig. 1(a). A gas layer on the top provides membrane valve control for zoospore sorting. The fluidic layer on the bottom is composed of parallel measurement channels, each containing a zoospore trap-site with a constriction structure in the entrance, shown in Fig. 1(b), and a freely bending elastomeric micropillar (diameter $7 \mu \mathrm{m}$, height $24 \mu \mathrm{m})$ as force sensor. Width and height of the measurement channels were 35 and $30 \mu \mathrm{m}$, respectively.

Zoospores are introduced from the zoospore inlet, flow along the loading channel and are captured in a trap site (Fig. 1(b)). Once all sites are occupied, the gas layer is used to actuate the membrane valve, which closes the channel and prevents trapped zoospores from escaping (Fig. 1(e)). The hyphae then grow through the measurement channels and deflect their respective force
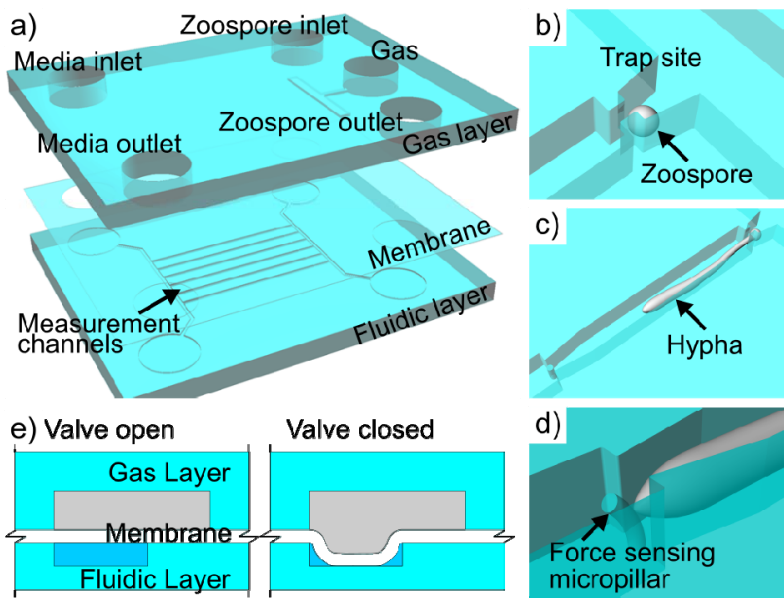

Figure 1: Concept of the lab-on-a-chip platform. (a) Entire chip showing the two-layer structure separated by a membrane. (b-d) Detailed view of the measurement channel while a zoospore is trapped, germinates and grows towards the force sensing pillar. (e) Cross-section of the membrane valve opening and closing the inlet channel on the fluidic layer. 
sensing pillars, as shown in Fig. 1(c) and (d). Meanwhile, media continues to be supplied from the media inlet to the end of the measurement channels, from where nutrients diffuse to the organisms.

\section{Platform fabrication}

Each PDMS layer was fabricated by replica-molding off photoresist masters and bonded via the central, spin-coated PDMS membrane. A two-layer photoresist master was fabricated for the fluidic layer by a combination of negative photoresist ADEX05 (DJ Microlaminates) and positive photoresist AZ 40XT (M.M.R.C Pty Ltd.), which provides a high-aspect freely-bending pillar inside a channel constriction [14].

For the gas layer master standard photolithography processing was used. In brief, a 4" chrome-on-glass photomask (Nanofilm) containing the gas layer pattern was prepared ( $\mu$ PG101, Heidelberg Instruments). Negative dry-film photoresist, ADEX 50 (thickness $50 \mu \mathrm{m}$, DJ Microlaminates) was laminated on a pre-cleaned 4" $\mathrm{Si}$ wafer. This was then exposed using UV lithography (MA-6, Suss) at an exposure dose of $325 \mathrm{~mJ} / \mathrm{cm}^{2}$ in vacuum contact mode with a filter (PL-360, Chroma). The wafer was developed in cyclohexanone, rinsed with isopropyl alcohol (IPA) and dried by $\mathrm{N}_{2}$, followed by a ramped post-exposure bake of $5 \mathrm{~min}$ at $65^{\circ} \mathrm{C}$ and $10 \mathrm{~min}$ at $95^{\circ} \mathrm{C}$. The master mold of the gas layer was completed by a hard-bake at $150^{\circ} \mathrm{C}$ for $1 \mathrm{~h}$.

Both PDMS fluidic and gas layers were molded into Sylgard 184 silicone elastomer (Dow Corning, 10:1 w/w). The two master molds were treated by vapor-coating with Trichloro $(1 \mathrm{H}, 1 \mathrm{H}, 2 \mathrm{H}, 2 \mathrm{H}$-perfluorooctyl)silane (TFOCS, Sigma-Aldrich) to facilitate mold release. Pre-mixed PDMS was poured onto the molds and degassed. After a $2 \mathrm{~h}$ bake at $80{ }^{\circ} \mathrm{C}$ on a hotplate, the PDMS layers were peeled off carefully and $1 \mathrm{~mm}$ holes punched for all the inlets and outlets. Meanwhile, pre-mixed (10:1 w/w) PDMS was spin-coated onto a TFOCS-treated Si wafer (WS-650, Laurell) at $3000 \mathrm{rpm}$ for $30 \mathrm{sec}$, achieving a thickness of $17 \mu \mathrm{m}$. The PDMS membrane was cured via $2 \mathrm{~h}$ bake at $80{ }^{\circ} \mathrm{C}$. Manual alignment, oxygen plasma bonding (Tergeo, PIE Scientific) and baking for $2 \mathrm{~h}$ at $80{ }^{\circ} \mathrm{C}$ completed the PDMS platform. Fabricated devices were degassed for $1 \mathrm{~h}$ in a vacuum chamber to improve filling with media and sealed into food-grade vacuum bags using a vacuum sealer (Sunbeam FoodSaver) for storage.

\section{Experimental setup}

A. bisexualis zoospores were produced through a starvation cycle of $A$. bisexualis [16]. In brief, six inoculum plugs from a fresh culture growing edge of $A$. bisexualis were evenly spread around on a nappy liner, which was placed on peptoneyeastglucose (PYG) agar Petrie dish. After incubating at $26^{\circ} \mathrm{C}$ for 24 hours, the nappy liner with the colony was peeled off and placed into a flask with PYG broth. Then it was swirled on an orbital shaker at $150 \mathrm{rpm}$ for 24 hours at $26{ }^{\circ} \mathrm{C}$. The PYG broth in the flask was exchanged with a mineral salt solution [16] six times and swirled again at $150 \mathrm{rpm}$ at $26^{\circ} \mathrm{C}$ overnight. The content of the flask was filtered by sterile Kimwipes and vortexed for $10 \mathrm{~s}$ to collect fully developed zoosporangia and released
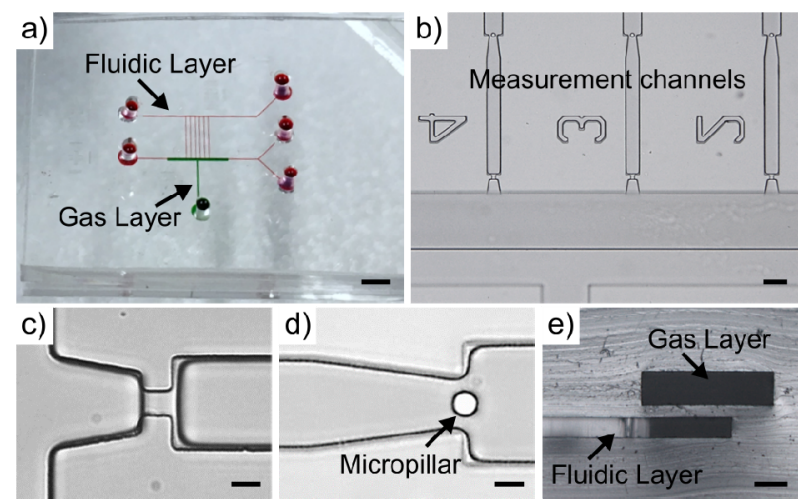

Figure 2: Fabricated PDMS platform. (a) Photograph of the entire platform filled with dye-colored water. (b-d) Micrographs of measurement channels, constriction structure and micropillar in a channel. (e) Micrograph showing a cross-section of the membrane valve. Scale bars are $2 \mathrm{~mm}, 50,10,10$ and $50 \mu \mathrm{m}$, respectively.

zoospores in solution. After centrifugation at $800 \mathrm{rpm}$ for $10 \mathrm{~min}, 20^{\circ} \mathrm{C}$, the supernatant was carefully removed and the zoospores were re-suspended in the mineral salt solution for storage before use.

The fluidic layer of PDMS platform was first filled with the mineral salt solution. Zoospores were then introduced from the zoospore inlet via syringe pump (NE-300, New Era Pump Systems) and hydrodynamically captured in the trap-sites [15]. Valves were then shut off, while PYG broth continued to be supplied from the other side of the measurement channels. During maintenance, both valve and media supply were driven by a pressure controller (OB1, Elveflow). Growth of hyphae from zoospores in the measurement channels and micropillar deflection by the tip of each hypha were recorded using an optical microscope (Eclipse 80i, Nikon). The force generated by individual hyphae was obtained by a combination of image processing in ImageJ (V1.51 h, FIJI) and analysis in MATLAB (2016a, Mathworks) $[12,14]$.

\section{RESULTS}

\section{Lab-on-a-chip device}

As shown in Fig. 2, high-aspect ratio, in-channel force sensing pillars were successfully integrated with hydrodynamic traps on a monolithic PDMS valving platform. The device currently contains six parallel measurement channels, each containing a trap and sensor pillar, while a single large valve closes off the zoospore inlet channel. Device fabrication was complicated by the fact that pillar integration requires channels with rectangular cross-section, while normally-open PDMS valves require rounded channel profiles. The positive resist layer required to integrate the pillars [14] currently precludes the use of resist reflow to form rounded structures for complete valve closure [15], as this would also distort the pillar shape. As such, the current design uses PDMS sieve valves to retain zoospores in the constriction structures.

\section{Trapping and compartmentalization}

To demonstrate trapping and compartmentalization on 

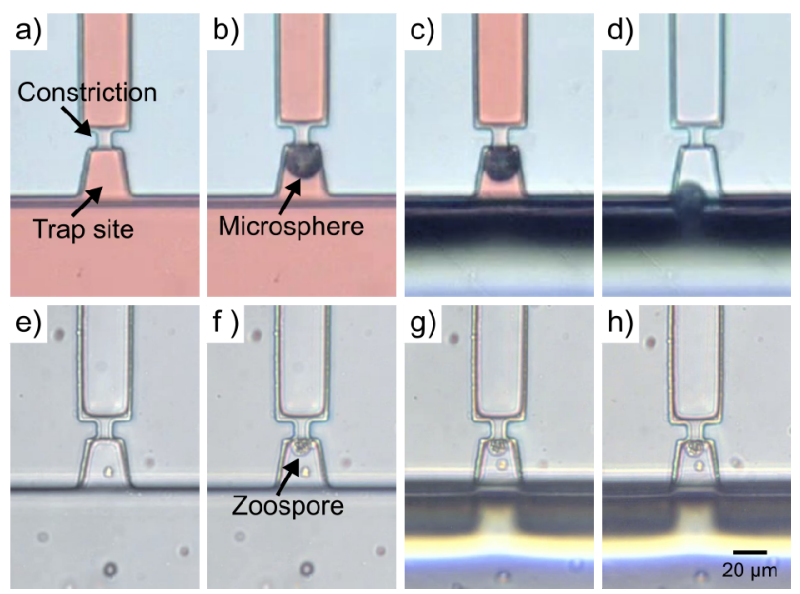

Figure 4: Trapping and maintenance functions of the platform demonstrated using polystyrene microspheres (20 $\mu \mathrm{m}$ diameter) and live A. bisexualis zoospores. (a) \& (b) Micrographs showing the constriction structure before and after a microsphere was captured in the trap site. (c) \& (d) Micrographs showing closure of the membrane valve and retaining of the microsphere after the red colored water was replaced by DI water. (e-h) Micrographs showing the initial trapping of a zoospore and successful retention during a media exchange cycle.

the platform, polystyrene microspheres $(20 \mu \mathrm{m}$ diameter $)$ suspended in water with red food coloring were first utilized. Figure 3 (a) and (b) show a single microsphere flowing into a measurement channel, closing of the constriction, and thus diverting successive microspheres to the next traps. After becoming trapped, the microsphere is retained in the trap site by closure of the membrane valve, as shown in Fig. 3 (c). DI water, provided to simulate media supply, faded the red color in the channel, but did not dislodge the microsphere (Fig. 3(d)). The same processes were applied to A. bisexualis zoospores, which range from 10 to $15 \mu \mathrm{m}$ in diameter. Figure 3 (e) to (f) show an example of the trapping and successful retention of a zoospore during a media exchange cycle.

Following trapping, the growth of hyphae germinating from zoospores compartmentalized into separate channels was observed using optical microscopy, as shown in Fig. 4. Two types of constrictions were tested, a Type A ( $8 \times 5 \mu \mathrm{m}$ width $\mathrm{x}$ height, additional vertical constriction) and a Type $\mathrm{B}(10 \times 30 \mu \mathrm{m}$ width $\times$ height, no vertical constriction), both based on previous work [15] and shown in Fig. 4(d). As indicated in Fig. 4(c), we observed that the growth success ratio of zoospores captured by the horizontal only constriction Type B was much higher ( 7 out of 10 zoospores observed) than that of horizontal and vertical constriction Type A ( 2 out of 12 zoospores observed). This may indicate a yet to be determined mechanosensory response by the zoospores to the trap geometry.

\section{Integrated force sensing}

Using the media exchange described above, trapped zoospores could be maintained on the platform until hyphae had germinated and extended to the force sensing pillars in their respective measurement channels. At this point, as described previously [12, 14], bright-field optical
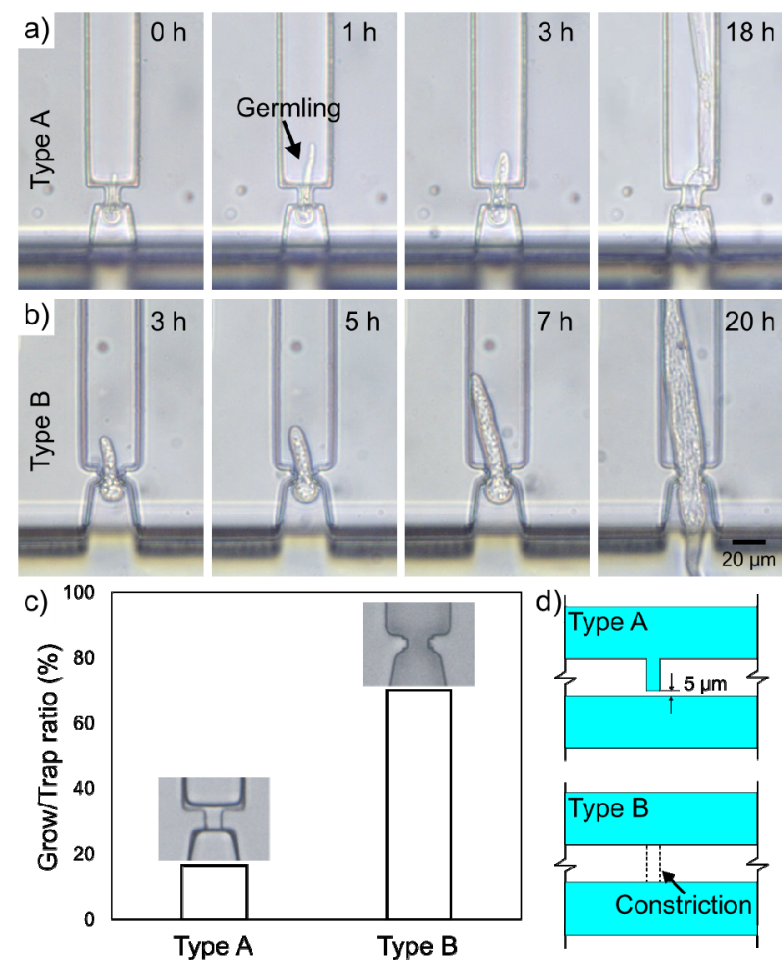

Figure 3: Growth of the A. bisexualis hyphae in the measurement channels with two types of constriction structure. (a) A sequence of micrographs showing a hypha growing from a germling through a combined horizontal and vertical constriction (Type A, $8 \times 5 \mu \mathrm{m}$ width $x$ height) after $0,1,3,18$ culture on the chip. (b) A sequence of micrographs showing a hypha growing through a horizontal-only constriction (Type $B, 10 \times 30 \mu \mathrm{m}$ width $\times$ height) after 3, 5, 7, $20 \mathrm{~h}$ culture. (c) Bar chart of growth success ratio for trapped zoospores with constriction Type $A$ and $B$. (d) Cross-section diagram of Type $A$ and $B$ showing the different heights of the constrictions.

microscopy and computational image processing techniques were used to measure the forces exerted by hyphae on the pillars. Figure 5 illustrates that both magnitude and direction of the force exerted by single hypha originating from single zoospores could be recorded as function of time. For the example hypha shown in Fig. 5(a), the force in $\mathrm{X}$ direction increased to $0.8 \mu \mathrm{N}$, while the force in Y direction fluctuated around $0.1 \mu \mathrm{N}$. As further illustrated by the plot of force versus time in Fig. 5(b), this significant increase in force perpendicular to the growth direction of this particular hyphae indicates a squeezing type event [12], where the hyphae is deflected into the gap between the pillar and channel wall and exerts force with the hyphal wall while traversing the gap.

Such squeezing type events predominantly occur for thinner hyphae and can be avoided by reducing the pillar-wall gap or growing hyphae to wider diameters [14]. We are currently investigating whether the position of the measurement pillar along the channel length can be used to tune the diameter of the hyphae. Previous work has indicated that, depending on the culture duration, hyphal length can be sensitive to channel length [15], but did not look at any correlation with channel width. In the future we 

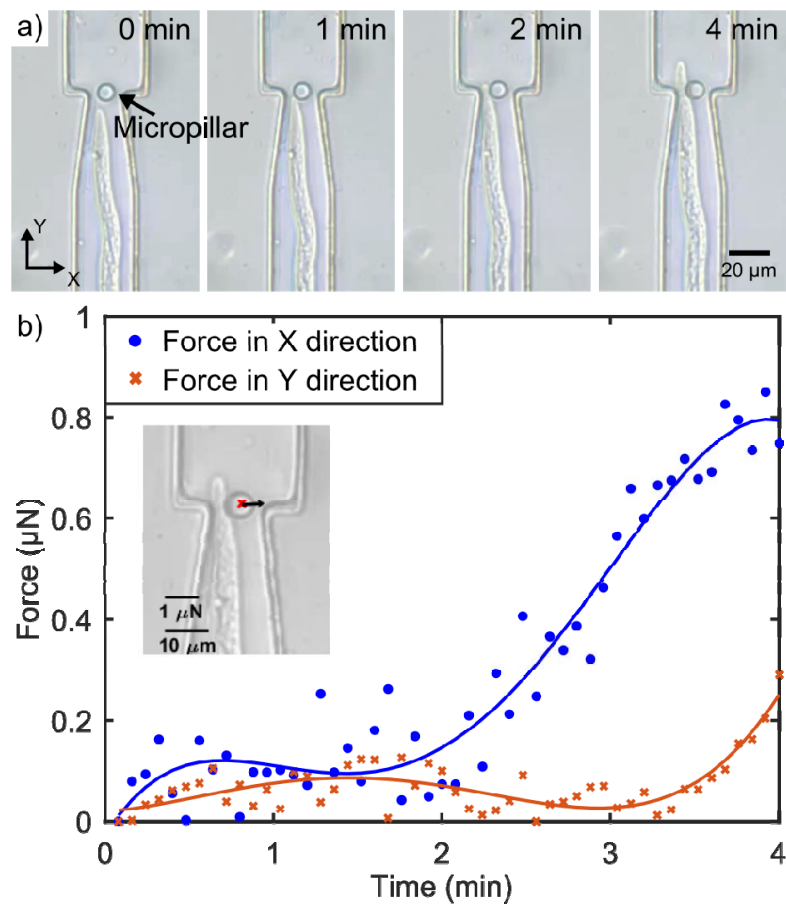

Figure 5: Force sensing on a hypha of A. bisexualis growing from an individual zoospore. (a) Sequence of micrographs showing the hypha growing towards a force sensing micropillar, hitting and deflecting it, and growing past it. (b) Plot of the force exerted by the hypha on the sensing pillar in both $X$ and $Y$ directions as function of time. Force in X-direction can be seen to increase significantly throughout the interaction, indicating a squeezing type event. The inset shows an example of the force vector overlay generated by the combined ImageJ and Matlab image analysis.

will use the platform to characterize the heterogeneity of force generation in populations of zoospores and to screen the effect of biocontrol compounds on the ability of hyphae to project protrusive force.

\section{CONCLUSIONS}

We have demonstrated the fabrication, characterization and application of a microfluidic platform combining in-channel, high-aspect ratio sensing pillars for protrusive force measurements on hyphal organisms with zoospore trapping, on-chip germination and organism maintenance. The platform constitutes a new tool to study heterogeneity in force generation by hyphae grown from individual zoospores, as well as screen biocontrol agents and strategies against populations of zoospores.

\section{ACKNOWLEDGEMENTS}

The authors would like to thank Helen Devereux and Gary Turner for technical assistance. Financial support was provided by Marsden Grant UOC1504, the Brian Mason Trust and the Biomolecular Interaction Centre.

\section{REFERENCES}

[1] M.C. Fisher et al., "Emerging fungal threats to animal, plant and ecosystem health." Nature, vol. 484, no. 7393, pp. 186-194, 2012.
[2] E. Pennisi, “Armed and dangerous.” Science, vol. 327, no. 5967, pp. 804-805, 2010.

[3] T. Giraud, P. Gladieux and S. Gavrilets, "Linking the emergence of fungal plant diseases with ecological speciation." Trends Ecol. Evol., vol. 25, no. 7, pp. 387-395, 2010.

[4] D.P. Bebber, M.A.T. Ramotowski and S.J. Gurr, "Crop pests and pathogens move polewards in a warming world." Nature Clim. Change, vol. 3, no. 11, pp. 985-988, 2013.

[5] M.C. Fisher et al., "Worldwide emergence of resistance to antifungal drugs challenges human health and food security." Science, vol. 360, no. 6390, pp. 739-742, 2018.

[6] A. Santini et al., "Tracing the role of human civilization in the globalization of plant pathogens." ISME J., vol. 12, no. 3, pp. 647-652, 2018.

[7] S. Farhana Syed Ab Rahman et al., "Emerging microbial biocontrol strategies for plant pathogens." Plant Sci., vol. 267, pp. 102-111, 2018.

[8] M. Bastmeyer, H.B. Deising and C. Bechinger, "Force exertion in fungal infection." Annu. Rev. Biophys. Biomol. Struct., vol. 31, no. 1, pp. 321-341, 2002.

[9] S.K. Walker et al., "Invasive hyphal growth: An F-actin depleted zone is associated with invasive hyphae of the oomycetes Achlya bisexualis and Phytophthora cinnamomi." Fungal Genet. Biol., vol. 43, no. 5, pp. 357-365, 2006.

[10] S. Suei and A. Garrill, "An F-actin-depleted zone is present at the hyphal tip of invasive hyphae of Neurospora crassa." Protoplasma, vol. 232, no. 3-4, pp. 165-172, 2008.

[11]A. Tayagui et al., "On-chip measurement of protrusive force exerted by single hyphal tips of pathogenic microorganisms." in Digest Tech. Papers MicroTAS Conference, Dublin, Ireland, Oct 9-13, pp. 150-151, 2016.

[12]A. Tayagui et al., "An elastomeric micropillar platform for the study of protrusive forces in hyphal invasion." Lab Chip, vol. 17, no. 21, pp. 3643-3653, 2017.

[13] Y. Sun et al., "A microfluidic platform with integrated sensing pillars for protrusive force measurements on Neurospora crassa." in Digest Tech. Papers MEMS Conference, Belfast, Northern Ireland, Jan 21-25, pp. 1116-1119, 2018.

[14] Y. Sun et al., "Fabrication of in-channel high-aspect ratio sensing pillars for protrusive force measurements on fungi and oomycetes." J. Microelectromech. Syst., vol. 27, no. 5, pp. 827-835, 2018.

[15] T. Geng et al., "Compartmentalized microchannel array for high-throughput analysis of single cell polarized growth and dynamics." Sci. Rep., vol. 5, $16111,2015$.

[16]A. Muralidhar et al., "A pressure gradient facilitates mass flow in the oomycete Achlya bisexualis." Microbiology, vol. 162, no. 2, pp. 206-213, 2016.

\section{CONTACT}

Yiling Sun: yiling.sun@.pg.canterbury.ac.nz 